\title{
EL TAMAÑO COMO ELEMENTO DETERMINANTE DE LA \\ INSOLVENCIA EN LAS SOCIEDADES COOPERATIVAS: \\ ESTUDIO A PARTIR DE LOS PROCESOS CONCURSALES
}

\author{
POR \\ Javier ITURRIOZ DEL CAMPO ${ }^{1}$ y \\ Sonia MARTÍN LÓPEZ ${ }^{2}$
}

\section{RESUMEN}

Con la crisis económica se ha incrementado el número de entidades que para intentar solucionar situaciones de insolvencia, antes de llegar a situación de liquidación y cierre, buscan una solución mediante el concurso de acreedores. En la primera parte de este trabajo se realiza un análisis de la evolución de las cooperativas que han iniciado un concurso de acreedores en el periodo 2005-2011. Posteriormente, se lleva a cabo un análisis del efecto del tamaño de las cooperativas tanto en las entidades concursadas como no concursadas utilizando la clasificación propuesta por la Unión Europea en función del número de empleados que diferencia entre microempresas, pequeñas empresas, y empresas de dimensión mediana y grande. Para ello se utilizan ratios económico-financieros, con el objetivo de lograr la adimensionialidad necesaria para evitar los efectos de las unidades de medida, y permitir estudiar la evolución del conjunto de empresas que constituyen la muestra a lo largo del período. Los ratios analizados se clasifican en cuatro grupos (estructura financiera, solvencia, liquidez, rentabilidad, y generación de recursos), aplicándolos tanto a las cooperativas concursadas, como a las no concursadas, y analizando la influencia de su tamaño.

\footnotetext{
${ }^{1}$ Javier Iturrioz del Campo: Universidad CEU San Pablo. Dirección de correo electrónico: itucam@ceu.es

${ }^{2}$ Sonia Martín López: Universidad Complutense de Madrid. Dirección de correo electrónico: soniamartinlopez@ccee.ucm.es
}

REVESCO No 111 - Segundo Cuatrimestre 2013 - ISSN: 1885-8031 - www.ucm.es/info/revesco

http://dx.doi.org/10.5209/rev_REVE.2013.v111.426753

Fecha de recepción: 25/02/2013

Fecha de aceptación: 05/04/2013 
Palabras clave: Concurso de acreedores, Sociedades Cooperativas, ratios económicofinancieros, insolvencia, tamaño

Claves ECONLIT: G33, J54, O16

\title{
SIZE AS A DETERMINANT OF INSOLVENCY IN COOPERATIVES: STUDY FROM MEETING OF CREDITORS
}

\begin{abstract}
With the economic crisis, the number of entities that try to solve insolvency situations, before being in liquidation and closing situation, and seek a solution in meeting of creditors, has increased. In the first part of this paper there is an analysis of the evolution of cooperatives which have initiated a meeting of creditors in the period 2005-2011. Subsequently, there is an analysis of the effect of the size of cooperatives. They are classified according to the proposal of the European Union based on the number of employees that difference among micro, medium-sized and large companies. Economic-financial ratios are used, to avoid the effects of measurement units, and to allow studying the evolution of the group of companies throughout the period. The ratios analyzed are classified into four groups (financial structure, solvency, liquidity, profitability, and generating resources). We focus on the analysis of both cooperatives societies that are in meeting of creditors, as those that are not in meeting of creditors, differing in both groups according to the size.
\end{abstract}

Key words: Meeting of creditors, cooperatives, economic - financial ratios, insolvency, size.

\section{INTRODUCCIÓN}

Desde mediados de la primera década del siglo XXI las repercusiones de la crisis económica han supuesto una creciente incapacidad de las empresas para hacer frente a sus acreedores. Esta situación afecta a todo tipo de empresas entre las que se incluyen las sociedades cooperativas, suponiendo un incremento de las entidades que inician un proceso de concurso de acreedores, con la esperanza de poder superar sus problemas y seguir ejerciendo su actividad. En este trabajo se analizan las peculiaridades de las sociedades cooperativas inmersas en este tipo de concursos, comparándolas con las cooperativas que se 
mantienen al margen de estos procesos, y estableciendo la comparativa tanto desde una perspectiva general, como diferenciando en función del tamaño de las cooperativas.

\section{EVOLUCIÓN DE LOS PROCESOS CONCURSALES DE LAS SOCIEDADES COOPERATIVAS.}

La falta de liquidez que se produce por la bajada de la actividad productiva de las empresas unida a la creciente dificultad para lograr recursos procedentes de las entidades financieras (GARCIA, MERINO y RUBIO, 2002), está produciendo una grave situación económico financiera para el tejido empresarial. Entre las alternativas para solucionar esta situación, el procedimiento concursal se presenta como un mecanismo legal que permite a las empresas con problemas de insolvencia buscar una solución a sus problemas. El objetivo de los sistemas concursales actuales consiste en buscar la continuidad de la empresa, y sólo en casos de inviabilidad establecen la liquidación de la entidad concursada (SOTO, 2010). A partir de la reforma concursal de 2003, se estableció un procedimiento único para solucionar las situaciones de insolvencia que anteriormente estaban divididas en la suspensión de pagos y la quiebra empresarial (ITURRIOZ, 2010; BELTRÁN, 2008).

El sistema concursal español se basa en las siguientes piezas legislativas:

- Ley Orgánica 8/2003, para la Reforma Concursal (LORC) $)^{3}$.

- $\quad$ Ley 22/2003, Ley Concursal (LC) ${ }^{4}$.

- Real Decreto Ley 3/2009, de medidas urgentes en materia tributaria, financiera y concursal (RDLMU) ${ }^{5}$.

- Ley 13/2009 de reforma de la legislación procesal para la implantación de la nueva oficina judicial (LRLP) ${ }^{6}$.

\footnotetext{
${ }^{3}$ ESPAÑA: LEY ORGÁNICA 8/2003, de 9 de julio, para la reforma concursal, BOE N. 164, de 10 de julio.

${ }^{4}$ ESPAÑA: LEY 22/2003, de 9 de julio, Concursal, BOE, N. 164, de 10 de julio de 2003.

5 ESPAÑA: REAL DECRETO LEY 3/2009, de 27 de marzo, de medidas urgentes en materia tributaria, financiera y concursal ante la evolución de la situación económica, BOE N. 78, de 31 de marzo. Con el que se reforma la LC buscando, entre otros aspectos: facilitar la refinanciación; agilizar los trámites; y reducir los costes del procedimiento.

${ }^{6}$ ESPAÑA: REAL DECRETO LEY 13/2009, de 3 de noviembre, de reforma de la legislación procesal para la implantación de la nueva Oficina Judicial, BOE N. 266, de 4 de noviembre. Esta norma modifica 49 apartados de la LC destacando los relacionados con: la descarga de tareas del juez concursal (traspasadas a la oficina
}

REVESCO No 111 - Segundo Cuatrimestre 2013 - ISSN: 1885-8031 - www.ucm.es/info/revesco 
El procedimiento establecido diferencia una primera fase, en la que se realiza la valoración de la situación de la empresa, determinando la continuidad de la empresa o la liquidación de la misma, que se producen en una segunda fase.

Este proceso se ha iniciado entre 2005 y 2007 por un número de empresas situado entre los 920 y 1.097 mostrando una reducción en el año 2006. En los años 2008 y 2009 el número de empresas en proceso concursal se multiplicó por más de siete, llegando a una cifra superior a las 5.800. Aunque en 2010 el número de empresas concursadas se redujo, en 2011 ha vuelto a aumentar alcanzando el valor máximo por encima de las 6.000 empresas concursadas (6.470).

Gráfico 1: Número de empresas concursadas

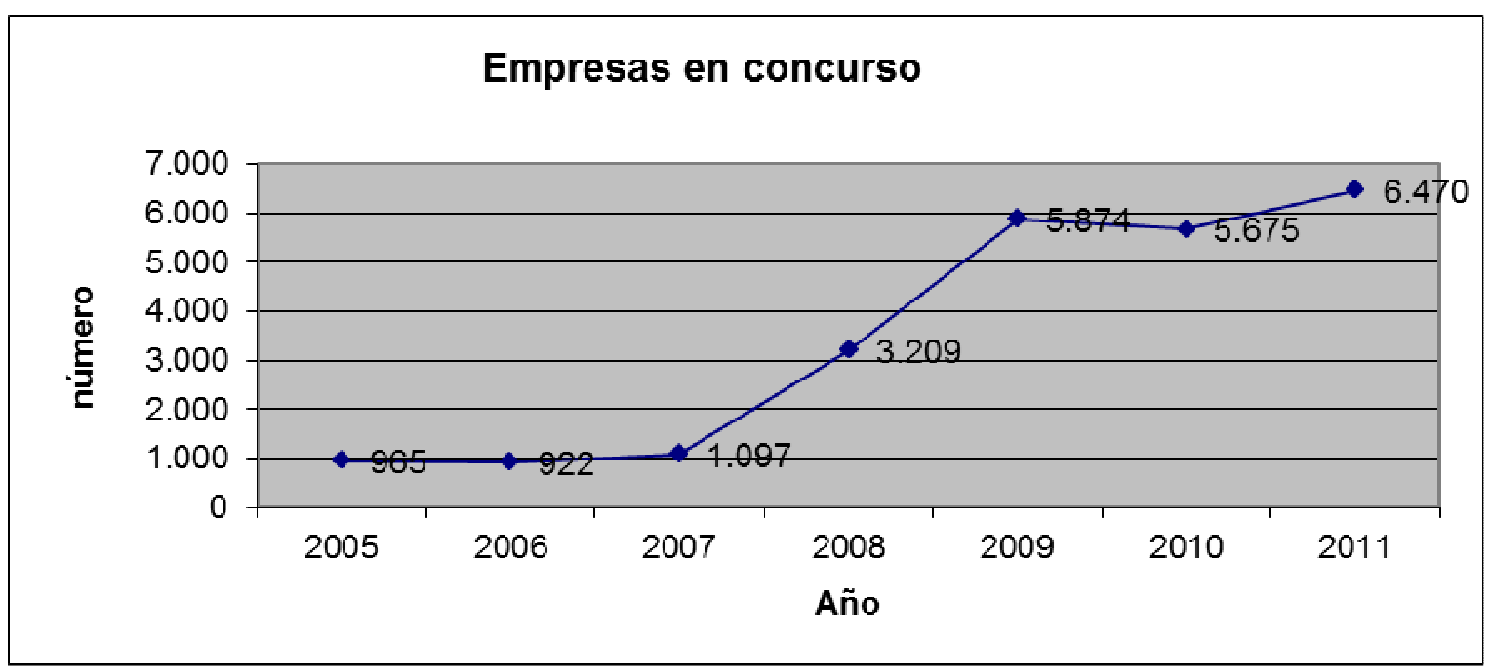

Fuente: Elaboración propia. Datos obtenidos del Boletín Oficial del Estado http://www.boe.es/diario_boel

Por su parte, el número de cooperativas concursadas presenta una evolución similar hasta 2009, con una pequeña reducción en 2006, y elevados aumentos en 2008 y 2009 (ITURRIOZ y MARTÍN, 2010). No obstante, muestra una tendencia diferente en 2010, ya que mientras el número total de empresas concursadas se ha reducido, el de cooperativas se ha incrementado. En 2011, se ha recuperado la tendencia alcista en el caso del conjunto de las empresas concursadas, y se ha mantenido la tendencia creciente mostrada por las sociedades cooperativas concursadas de los años anteriores, aunque en este último año la pendiente es mayor. El peso porcentual de las cooperativas con respecto al total de empresas concursadas 
se mantuvo, en el período 2005 y 2007 , entre el 0,87 por ciento y el 1 por ciento. Sin embargo, en 2008 y 2009 su peso se ha reducido a valores cercanos al 0,5 por ciento mientras que en 2010 se vuelve a incrementar hasta el 0,72 por ciento, y en el año 2011 dicho porcentaje se sitúa en el 1,08 por ciento.

Gráfico 2: Número de cooperativas concursadas

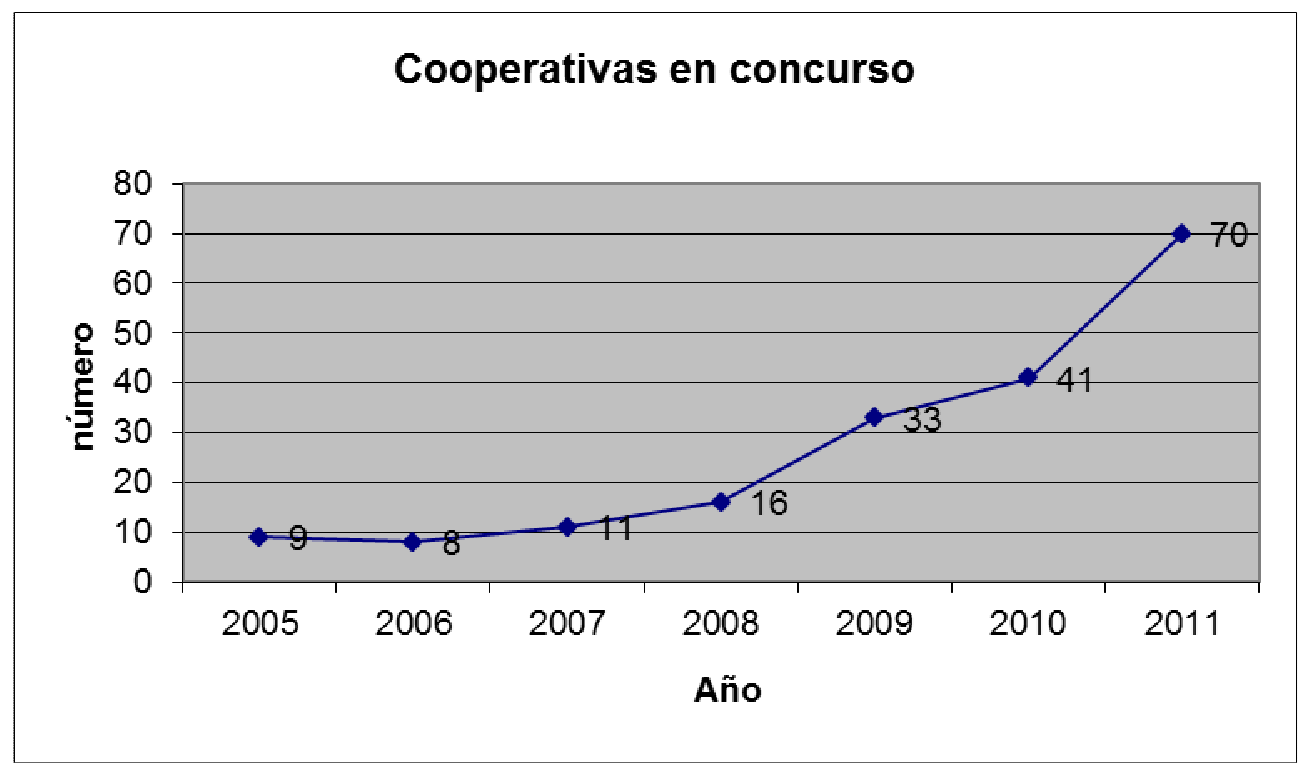

Fuente: Elaboración propia. Datos obtenidos del Boletín Oficial del Estado http://www.boe.es/diario_boe/

Analizando la tendencia de la evolución porcentual se observa que, desde 2007 a 2009, se han producido incrementos porcentuales tanto en el total de empresas como en el número de cooperativas mientras que en el 2010 el incremento sólo se mantiene en las cooperativas. No obstante, en el año 2011 se vuelve a recuperar la tendencia mostrada en los años anteriores. En el total de empresas destaca el incremento del 193 por ciento en el 2008, y del 83 por ciento en 2009 (esta última cifra indica un aumento del 1.422 por ciento con respecto a 2007) aunque la reducción porcentual del 3 por ciento en 2010 podría haber marcado un cambio de tendencia. No obstante, los datos del 2011 no confirman dicho cambio de tendencia, dado que el número de empresas concursadas en relación con las existentes en el año 2010 se incrementó en un 14 por ciento. En el caso de las sociedades cooperativas, en 2008 el aumento porcentual fue más reducido (un 45 por ciento frente a un 193 por ciento), aunque desde entonces el porcentaje de incremento en este tipo de entidades supera al del total de empresas. Por su parte, en 2010 el porcentaje incrementado es más reducido (pasa de un 106 a un 24 por ciento), sin embargo, en el año 2011 el incremento se sitúa en el 71 por 
ciento. Si se analiza la tendencia se puede observar que la reducción en el incremento porcentual del total de empresas se produjo en 2008, alcanzando un incremento máximo del 193 por ciento. Por su parte el cambio de tendencia en las sociedades cooperativas ha llegado un año más tarde. No obstante, las sociedades cooperativas concursadas no han reflejado la reducción que tuvo lugar en el año 2010 en el conjunto de empresas concursadas.

Gráfico 3: Incrementos porcentuales

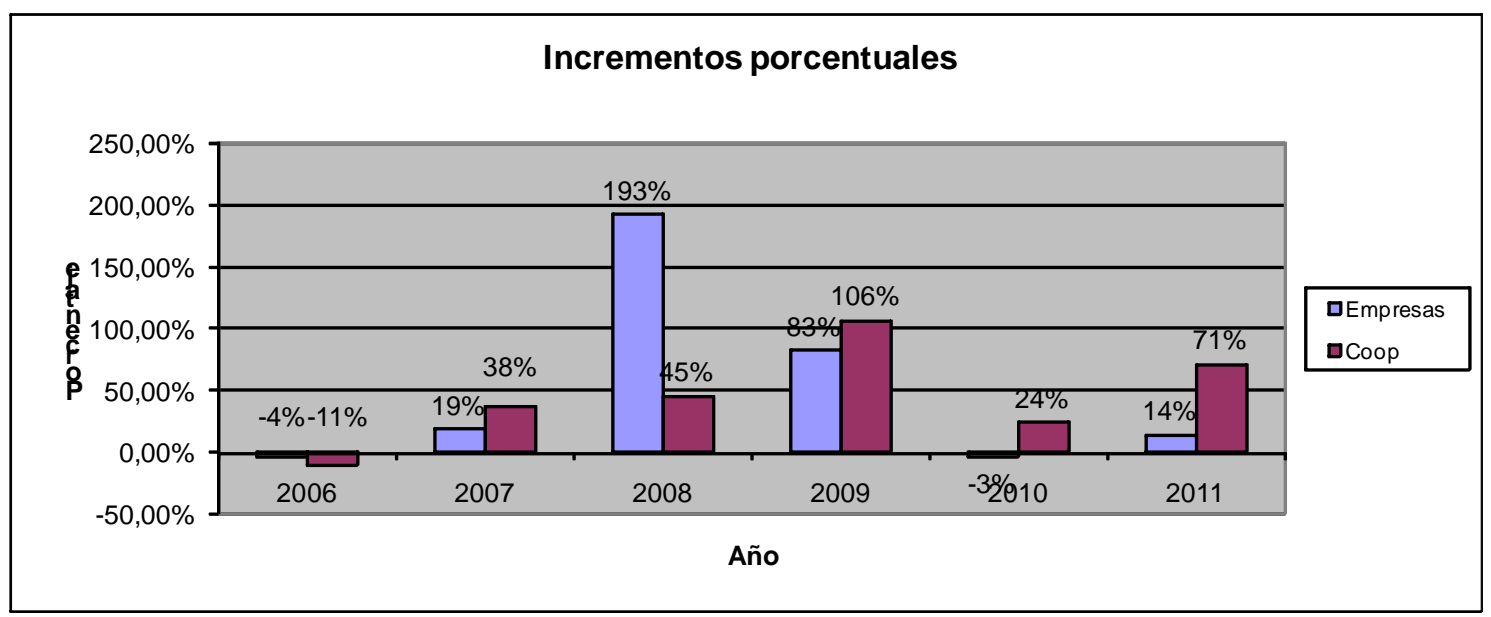

Fuente: Elaboración propia. Datos obtenidos del Boletín Oficial del Estado http://www.boe.es/diario_boe/

\section{COMPARACIÓN DE LAS CARACTERÍSTICAS ECONÓMICO-FINANCIERAS DE LAS SOCIEDADES COOPERATIVAS CONCURSADAS Y NO CONCURSADAS.}

El estudio se realiza tomando como referencia el total de las sociedades cooperativas, diferenciando entre aquellas que han entrado en un concurso de acreedores, entre los años 2005 y 2011, y las que no lo han hecho. Las cooperativas incluidas en el primer grupo se han obtenido del Boletín Oficial del Estado, obteniendo los datos económico - financieros de ambos grupos de la base de datos SABI (Sistema de Análisis de Balances Ibéricos). Los datos empleados corresponden a los del último año disponible, utilizando en el caso de las cooperativas concursadas el anterior a su entrada en el concurso. La muestra utilizada está formada por 2.968 cooperativas de las que 58 corresponden a cooperativas inmersas en un concurso de acreedores. En el análisis de la información económico financiera extraída de los estados contables de las sociedades cooperativas analizadas hay que tener en cuenta las particularidades de este tipo de sociedades (GARCÍA-GUTIÉRREZ, 2006; CASANELLAS, 2009; MATEOS, 2008). 
Para poder identificar las posibles diferencias derivadas del tamaño de las cooperativas, se ha optado por realizar un análisis diferenciado tomando como referencia la clasificación propuesta por la Unión Europea en función del número de empleados ${ }^{7}$. Así, del total de la muestra; el 66 por ciento son microempresas, el 22 por ciento son pequeñas empresas, y el 12 por ciento son empresas de dimensión mediana y grande.

La metodología utilizada es el análisis de ratios, al considerarse adecuada para llevar a cabo el estudio económico-financiero de estas empresas, dado que proporciona la adimensionilidad necesaria para evitar los efectos de las unidades de medida, y permite estudiar la evolución del conjunto de empresas que constituyen la muestra a lo largo del período. El estudio se ha llevado a cabo haciendo un análisis diferenciado teniendo en cuenta los siguientes aspectos: estructura financiera, solvencia, liquidez, rentabilidad, y generación de recursos.

\section{A) Estructura financiera.}

Para analizar la estructura financiera de las sociedades cooperativas, y conocer la distribución del patrimonio neto y del pasivo, se utilizan los siguientes ratios:

- Ratio de endeudamiento: el objetivo es analizar la distribución de los recursos financieros en la sociedad cooperativa entre fondos propios y ajenos, y en concreto determinar el peso que tienen los recursos ajenos sobre la totalidad de los recursos financieros disponibles en la sociedad cooperativa, pudiéndose utilizar como una medida del riesgo financiero en la misma. Se calcula como:

$$
\text { Ratio de endeudamiento }=\frac{\text { Pasivo Exigible }}{\text { Patrimonio Neto y Pasivo }}
$$

En las sociedades cooperativas, hay que tener en cuenta la particularidad de que la cifra de capital social es un pasivo exigible ${ }^{8}$ dado que el socio puede

\footnotetext{
${ }^{7}$ Según la actualización de la definición de microempresas, pequeñas y medianas empresas, con el objetivo de reflejar mejor su realidad económica, recogida en la Recomendación 2003/361/CE de la Comisión, de 6 de mayo de 2003, sobre la definición de microempresas, pequeñas y medianas empresas [Diario Oficial L 124 de 20.5.2003], se establece que en función del número de empleados, serán microempresas aquellas que tengan menos de 10 trabajadores, empresas pequeñas las que tengan entre 10 y 49 trabajadores, empresas medianas las que tengan entre 50 y 249 trabajadores, y grandes empresas las que tengan 250 , o más, trabajadores.

${ }^{8}$ El criterio delimitador para que el capital social sea considerado pasivo exigible, o pueda ser considerado dentro de los fondos propios, lo constituye fundamentalmente la obligación frente al socio de la cooperativa, tanto de devolución del capital aportado como de pago de una remuneración. ESPAÑA. Orden EHA/3360/2010,
}

REVESCO No 111 - Segundo Cuatrimestre 2013 - ISSN: 1885-8031 - www.ucm.es/info/revesco 
solicitar su reembolso en caso de baja (MARTÍN, LEJARRIAGA e ITURRIOZ, 2007; GARCÍA-GUTIÉRREZ, 1987). Señalar, además, que las sociedades cooperativas han de dotar el Fondo de Reserva Obligatorio 9 (ITURRIOZ, 1999; ITURRIOZ, 1998).

En términos generales se puede decir que las sociedades cooperativas no concursadas presentan una menor dependencia de los recursos ajenos que se cuantifica, en media en un 4 por ciento. Por tamaños, las microempresas son las que presentan un menor peso del pasivo exigible, siendo las microempresas no concursadas las únicas con un valor medio inferior a 0,9 . Tanto en las microempresas como en las pequeñas cooperativas, las no concursadas presentan un valor inferior del ratio, aunque sorprende el hecho de que en las medianas y grandes, las concursadas tienen la misma dependencia de los recursos financieros ajenos, lo que indica que la dependencia de la financiación ajena es más elevada en las cooperativas de pequeño tamaño, especialmente en las concursadas.

Tabla 1: Ratio de Endeudamiento de las cooperativas concursadas y no concursadas

\begin{tabular}{|l|c|c|c|}
\cline { 2 - 4 } \multicolumn{1}{c|}{} & Coop. no concursada & Coop. concursada & Diferencia \\
\hline Microempresa & 0,88 & 0,91 & $-0,03$ \\
\hline Pequeña & 0,92 & 0,97 & $-0,05$ \\
\hline Mediana y Grande & 0,93 & 0,93 & 0,00 \\
\hline Total & 0,90 & 0,94 & $-0,04$ \\
\hline
\end{tabular}

Fuente: Elaboración propia. Nota: Datos calculados como valores medios

- Ratio de capitales propios: cuanto mayor sea el peso que tienen los capitales propios sobre los capitales permanentes en la sociedad cooperativa, menor será el riesgo financiero de la misma (MARTÍN, LEJARRIAGA, ITURRIOZ, 2007), al reducirse en la misma la dependencia de la financiación ajena. Se calcula como:

$$
\text { Ratio de capitales propios }=\frac{\text { Fondos Propios }}{\text { Capitales Permanentes }}
$$

de 21 de diciembre, por la que se aprueban las normas sobre los aspectos contables de las sociedades cooperativas. Boletín Oficial del Estado, no 316, de 29 de diciembre.

${ }^{9}$ El Fondo de Reserva Obligatorio se calificará como patrimonio neto o como pasivo en función de si es exigible. Íbidem.

REVESCO No 111 - Segundo Cuatrimestre 2013 - ISSN: 1885-8031 - www.ucm.es/info/revesco 
La evolución del ratio de capitales propios confirma los resultados expuestos anteriormente, ya que las cooperativas no concursadas presentan, en media, un mayor peso de los recursos propios en el total de las fuentes financieras a largo plazo. Por tamaño, se observa que a medida que aumenta la dimensión de la empresa mejora el ratio, aunque la diferencia entre concursadas y no concursadas se mantiene en un 10 por ciento en el caso de microempresas y pequeñas cooperativas. Para el caso de las cooperativas medianas y grandes la diferencia entre las concursadas y no concursadas se incrementa hasta el 34 por ciento, lo que pone de manifiesto que las diferencias entre la estructura financiera de las cooperativas concursadas se hace especialmente significativa para aquellas que tienen una dimensión superior.

Tabla 2: Ratio de Capitales Propios de las sociedades cooperativas concursadas y no concursadas

\begin{tabular}{|l|c|c|c|}
\cline { 2 - 4 } \multicolumn{1}{c|}{} & Coop. no concursada & Coop. concursada & Diferencia \\
\hline Microempresa & 0,49 & 0,48 & 0,01 \\
\hline Pequeña & 0,50 & 0,49 & 0,01 \\
\hline Mediana y Grande & 0,57 & 0,22 & 0,34 \\
\hline Total & 0,50 & 0,42 & 0,08 \\
\hline
\end{tabular}

Fuente: Elaboración propia. Nota: Datos calculados como valores medios.

\section{B) Análisis de solvencia.}

La solvencia determina la capacidad de la sociedad cooperativa para hacer frente a los pagos relativos a su nivel de endeudamiento, es decir, a la financiación ajena. Los ratios utilizados son el que mide la relación entre el total del activo y los recursos ajenos, así como el denominado ratio de capacidad de devolución.

- Ratio de solvencia. Tiene en cuenta el grado de cobertura del activo total en relación con el pasivo exigible, con lo que se analiza la distancia a la quiebra técnica (VARGAS, 2008). Lo deseable es que este ratio tome valores superiores a la unidad, dado que, cuanto mayor sea el valor del ratio más solvente será la sociedad cooperativa. 


$$
\text { Ratio de solvencia }=\frac{\text { Activo Total }}{\text { Pasivo Exigible }}
$$

Así, del análisis de la solvencia de las sociedades cooperativas objeto de estudio, se desprende que las no concursadas presentan una mayor solvencia en media. Centrándose en las cooperativas no concursadas se observa que cuanto mayor es el tamaño menor es su solvencia. Sin embargo, en el grupo de las concursadas, hay coincidencia en que las microempresas ofrecen el mejor ratio, pero las pequeñas se muestran más solventes que las medianas y grandes. Estudiando las diferencias entre grupos, son las últimas la que presentan una mayor discrepancia.

Tabla 3: Ratio de Solvencia de las cooperativas concursadas y no concursadas

\begin{tabular}{|l|c|c|c|}
\cline { 2 - 4 } \multicolumn{1}{c|}{} & Coop. no concursada & Coop. concursada & Diferencia \\
\hline Microempresa & 1,65 & 1,21 & 0,43 \\
\hline Pequeña & 1,49 & 1,01 & 0,48 \\
\hline Mediana y Grande & 1,40 & 1,10 & 0,30 \\
\hline Total & 1,58 & 1,08 & 0,50 \\
\hline
\end{tabular}

Fuente: Elaboración propia. Nota: Datos calculados como valores medios.

- Ratio de capacidad de devolución: Medido en función de la capacidad de devolución de la financiación ajena. Un valor elevado de este ratio indica un empeoramiento en la capacidad de devolución de los recursos ajenos.

$$
\text { Ratio de capacidad devolucion }=\frac{(\text { Pasivo Exigible LP }+ \text { Pasivo Exigible CP })}{(\text { Importe neto de la cifra ventas }+ \text { Dot amortización })}
$$

Tabla 4: Ratio de Capacidad de Devolución de las cooperativas concursadas y no concursadas

\begin{tabular}{|l|c|c|c|}
\cline { 2 - 4 } \multicolumn{1}{c|}{} & Coop. no concursada & Coop. concursada & Diferencia \\
\hline Microempresa & 0,80 & 1,27 & $-0,47$ \\
\hline Pequeña & 0,82 & 1,11 & $-0,28$ \\
\hline Mediana y Grande & 0,57 & 0,54 & 0,03 \\
\hline Total & 0,78 & 0,98 & $-0,20$ \\
\hline \multicolumn{2}{|c|}{ Fuente: Elaboración propia. Nota: Datos calculados como valores medios. }
\end{tabular}

Las cooperativas no concursadas muestran, en media, un mejor ratio de capacidad de devolución con una diferencia de 0,2 sobre las concursadas, lo que está en concordancia con lo mostrado en el ratio anterior. Sin embargo, por tamaños, las que presentan una menor capacidad para hacer frente a la financiación ajena son las micro cooperativas, con un ratio de 
1,27. A su vez este tipo de empresas es la que muestra una mayor diferencia $(0,47)$ con respecto a las no concursadas. En la misma línea, pero con un mejor ratio, aparecen las cooperativas pequeñas, en las que la diferencia con las no concursadas se queda en 0,28. En cuanto a las de mayor tamaño, el ratio medio es mucho más reducido, tanto en las concursadas como en las no concursadas, lo que puede obedecer a una mayor dotación de amortizaciones derivada de su mayor inmovilizado, mostrando las primeras un valor mas reducido en media. Por tanto, a la vista de estos datos, la capacidad para la devolución de los recursos ajenos muestra una relación directa con el tamaño de la cooperativa, lo que no coincide con lo analizado en el ratio anterior. El motivo puede encontrarse en que mientras en el ratio de solvencia se mide la relación entre recursos ajenos y activo, en el de capacidad de devolución se analiza la capacidad para obtener recursos líquidos gracias a las ventas y a la dotación de amortizaciones.

\section{C) Análisis de liquidez.}

La liquidez a corto plazo de la sociedad cooperativa analiza la capacidad de hacer frente a los pagos relativos al ciclo de explotación. Cuanto mayor sea su valor, menos dificultades tendrá la sociedad cooperativa para pagar las deudas de vencimiento a corto plazo.

$$
\text { Ratio de liquidez }=\frac{(\text { Exigible }+ \text { Disponible })}{\text { Pasivo Exigible a corto plazo }}
$$

Las cooperativas no concursadas también muestran una mayor liquidez en media con una diferencia de 0,55. Dentro de las concursadas son las microempresas las que ofrecen mejores ratios mientras que las pequeñas no llegan a la unidad. Sin embargo, en las no concursadas las medianas son las que muestran una mejor situación mientras que la microempresas y las pequeñas ofrecen un ratio similar (MARTÍN, LEJARRIAGA, y GARCÍA-GUTIÉRREZ, 2010). Si se comparan los dos grupos, cuanto mayor es el tamaño, más amplia es la diferencia. 
Tabla 5: Ratio de Liquidez de las cooperativas concursadas y no concursadas

\begin{tabular}{|l|c|c|c|}
\cline { 2 - 4 } \multicolumn{1}{c|}{} & Coop no concursada & Coop concursada & Diferencia \\
\hline Microempresa & 1,62 & 1,34 & 0,28 \\
\hline Pequeña & 1,65 & 0,99 & 0,67 \\
\hline Mediana y Grande & 1,86 & 1,08 & 0,79 \\
\hline Total & 1,66 & 1,11 & 0,55 \\
\hline
\end{tabular}

Fuente: Elaboración propia. Nota: Datos calculados como valores medios.

\section{D) Análisis de la Rentabilidad.}

Para analizar la rentabilidad de las cooperativas que componen la muestra se utilizan los ratios de rentabilidad económica y financiera.

- La rentabilidad económica mide la capacidad de la sociedad cooperativa para retribuir a los capitales invertidos. Cuanto mayor sea el valor de dicho ratio, más rentable será la cooperativa al ser capaz de generar mayores beneficios dada su estructura económica.

$$
\text { Ratio de rentabilidad económica }=\frac{\text { Resultado Neto Explotación }}{\text { Activo Total }}
$$

La rentabilidad económica de las cooperativas no concursadas muestra mejores valores que las de las concursadas. Hay que mencionar que en el grupo de no concursadas, tanto las micro cooperativas como las pequeñas empresas presentan rentabilidades negativas, lo que pone de manifiesto la no consecución de excedentes positivos. En cuanto a los tamaños, en ambos grupos, se muestra una mayor rentabilidad económica para las medianas y grandes empresas y un valor más reducido para las pequeñas.

Tabla 6: Ratio de Rentabilidad Económica de las cooperativas concursadas y no concursadas

\begin{tabular}{|l|c|c|c|}
\cline { 2 - 4 } \multicolumn{1}{c|}{} & Coop. no concursada & Coop. concursada & Diferencia \\
\hline Microempresa & 0,14 & $-0,56$ & 0,70 \\
\hline Pequeña & 0,08 & $-0,84$ & 0,92 \\
\hline Mediana y Grande & 0,52 & 0,42 & 0,09 \\
\hline Total & 0,16 & $-0,47$ & 0,64 \\
\hline
\end{tabular}

Fuente: Elaboración propia. Nota: Datos calculados como valores medios.

- La rentabilidad financiera mide la capacidad de la sociedad cooperativa para retribuir a los socios. Cuanto mayor sea el valor de dicho ratio, mayores serán los recursos que la empresa podrá destinar bien a retribuir a los socios, o la 
dotación de reservas. En el caso de las sociedades cooperativas la retribución de los socios tiene algunas particularidades ya que la retribución por las aportaciones sociales de los socios se realiza antes de impuestos, mientras que el resultado final se utiliza para retribuir la participación de los socios en la obtención del resultado de la cooperativa y no su aportación financiera.

$$
\text { Ratio de rentabilidad financiera }=\frac{\text { Resultado del Ejercicio }}{\text { Fondos Propios }}
$$

Tabla 7: Rentabilidad Financiera de las cooperativas concursadas y no concursadas

\begin{tabular}{|l|c|c|c|}
\cline { 2 - 4 } \multicolumn{1}{c|}{} & Coop. no concursada & Coop. concursada & Diferencia \\
\hline Microempresa & 7,04 & 0,83 & 6,21 \\
\hline Pequeña & 5,11 & 0,49 & 4,62 \\
\hline Mediana y Grande & 12,70 & 5,59 & 7,11 \\
\hline Total & 7,16 & 1,72 & 5,44 \\
\hline
\end{tabular}

Fuente: Elaboración propia. Nota: Datos calculados como valores medios.

El análisis de la rentabilidad financiera es similar al de la rentabilidad económica, mostrando un mayor ratio para las no concursadas. Si se realiza el análisis en función del tamaño vuelve a ponerse de manifiesto una mayor rentabilidad financiera para las cooperativas medianas y grandes (ITURRIOZ, 1998), y unos ratios más reducidos para las pequeñas. Sin embargo, a diferencia de lo que ocurre en la rentabilidad económica, la diferencia más amplia se encuentra en las medianas y grandes.

\section{E) Generación de recursos.}

Mediante el ratio de generación de recursos, se ponen en relación los recursos generados por la sociedad cooperativa en el ejercicio con respecto a los recursos ajenos a corto plazo. De esta forma se determina el número de veces que está incluido el pasivo exigible a corto plazo en los recursos generados por la empresa, es decir, el número de veces que sería capaz la empresa de devolver las deudas contraídas con terceros con vencimiento inferior al año. Cuanto mayor sea este ratio menores serán los problemas de atención al pago por parte de la sociedad cooperativa al vencimiento de las deudas en el corto plazo.

$$
\text { Ratio de generación de recursos }=\frac{(\text { Rdo Neto Explotación }+ \text { Dot amortización })}{\text { Pasivo Exigible a Corto Plazo }}
$$


El ratio de generación de recursos está en línea con lo expuesto en los párrafos anteriores, ya que muestra una mejor situación en las cooperativas que no están inmersas en un concurso de acreedores. Las micro cooperativas son las que presentan una mayor capacidad de generación de recursos dentro de las concursadas, mientras que son las peores en las no concursadas (ITURRIOZ, 1999). Sin embargo, son las que presentan una menor diferencia entre concursadas y no concursadas.

Tabla 8: Ratio de Generación de Recursos de las cooperativas concursadas y no concursadas

\begin{tabular}{|l|c|c|c|}
\cline { 2 - 4 } \multicolumn{1}{c|}{} & Coop. no concursada & Coop. concursada & Diferencia \\
\hline Microempresa & 0,23 & 0,13 & 0,10 \\
\hline Pequeña & 0,34 & 0,03 & 0,31 \\
\hline Mediana y Grande & 0,34 & 0,07 & 0,27 \\
\hline Total & 0,27 & 0,06 & 0,20 \\
\hline
\end{tabular}

Fuente: Elaboración propia. Nota: Datos calculados como valores medios.

\section{CONCLUSIONES}

En media, las cooperativas no inmersas en un proceso concursal presentan mejores valores en todos los ratios analizados. No obstante, hay que mencionar algunas diferencias relacionadas con el tamaño de las cooperativas. Centrándonos en las cooperativas concursadas se observan los siguientes aspectos:

Por lo que respecta a la estructura financiera, son las microempresas concursadas las que presentan una menor dependencia de los recursos ajenos, siendo las de dimensión pequeña las que ofrecen una dependencia superior a la vez que ofrecen las mayores discrepancias con respecto a las no concursadas. Sin embargo, si se tiene en cuenta el ratio de capitales propios, son las pequeñas las que muestran un mayor peso de los recursos propios con respecto al total de las fuentes financieras a largo plazo, lo que permite una reducción de su riesgo financiero. La explicación puede deberse a que este tipo de empresas tengan una mayor dotación a los distintos fondos obligatorios, para lo que es importante ser conscientes de las particularidades de los fondos de reserva de estas entidades, especialmente del fondo de reserva obligatorio y del fondo de educación y promoción. Las cooperativas medianas y grandes son las que presentan un peor ratio y una mayor divergencia con respecto a las no concursadas. 
En cuanto a la solvencia los tres grupos presentan, en media, un ratio superior a la unidad, siendo las cooperativas de tamaño pequeño las que mejor situación presentan en cuanto a la quiebra técnica y las que cuentan con un peor ratio de capacidad de devolución. Si se tiene en cuenta el último ratio, la capacidad para la devolución de los recursos ajenos muestra una relación directa con el tamaño de la cooperativa, lo que no coincide con lo analizado en el ratio anterior. El motivo puede encontrarse en que mientras en el ratio de solvencia se mide la relación entre recursos ajenos y activo, en el de capacidad de devolución se analiza la capacidad para obtener recursos líquidos gracias a las ventas y a la dotación de amortizaciones, que suele ser más elevada en las empresas de mayor tamaño por su mayor inmovilizado amortizable.

Las micro cooperativas son, dentro de las concursadas, las que presentan una mayor liquidez a corto plazo, siendo las cooperativas de pequeño tamaño las que ofrecen un ratio más reducido, siendo las únicas con un valor inferior a la unidad. Las medianas y grandes, se encuentran en una situación intermedia a pesar de ser las que más diferencias presentan con respecto a las no concursadas.

En la rentabilidad, tanto económica como financiera, son las cooperativas de mayor dimensión las que presentan mejores ratios, mientras que, en ambos casos, las pequeñas aparecen con los peores datos. Hay que mencionar los valores negativos en las cooperativas pequeñas y en las micro cooperativas en cuanto a la generación de recursos por su estructura económica. La diferencia con las de mayor tamaño, en media, es muy elevada, destacando su rentabilidad financiera. Al compararlas con las no concursadas se observa una estructura similar, siendo las de mayor tamaño las que muestran más diferencias a la hora de poder destinar recursos a la retribución de los socios o la dotación de reservas. En este sentido hay que tener en cuenta las peculiaridades de las sociedades cooperativas tanto en la retribución financiera de los socios como en la dotación de fondos de reserva.

El ratio de generación de recursos muestra una situación similar a la de la liquidez, en la que son las microempresas las que muestran más capacidad para la devolución de sus deudas a corto plazo con los recursos generados, y las cooperativas de pequeño tamaño, las que ofrecen un peor ratio. La diferencia se encuentra al compararlas con las no concursadas, ya que mientras en la liquidez las que mostraban una mayor discrepancia eran las de mayor tamaño, en este caso son las de pequeña dimensión. 
Puede concluirse analizando de forma conjunta los ocho ratios utilizados en los que se muestra que no hay un tamaño de empresa que muestre una mejor situación de forma recurrente. Así, las microempresas presentan los mejores valores en tres (endeudamiento, liquidez y generación de recursos), las pequeñas en dos (capitales propios y solventa), mientras que las medianas y grandes lo hacen en los otros tres (capacidad de devolución, rentabilidad económica y rentabilidad financiera). Sin embargo, las cooperativas pequeñas son las que presentan un peor valor para seis de lo ocho ratios, todos excepto capitales propios, (medianas y grandes), y solvencia (micro cooperativas). Por último, señalar que al compararlas con las no concursadas vuelven a ser las pequeñas las que ofrecen mayores divergencias (en cuatro ratios; endeudamiento, solvencia, rentabilidad económica y generación de recursos), seguidas por las medianas y grandes con tres aspectos (liquidez, rentabilidad financiera y capitales propios), mientras que las micro cooperativas sólo aparecen con la mayor disparidad en el apartado de capitales permanentes.

\section{BIBLIOGRAFÍA}

BELTRAN, E.: Esquemas de derecho concursal, Tirant Loblanch, Valencia, 2008, ISBN: 978-84-9876-354-6.

CASANELLAS BASSOLS, R.: El nuevo plan general de contabilidad y el concurso de acreedores, Revista de derecho concursal y paraconcursal: Anales de doctrina, praxis, jurisprudencia y legislación, $\mathrm{n}^{\circ} .11,2009$, p. 395-401

GARCÍA TABUENCA, A., MERINO, F. y RUBIO, D.: Financiación de la pequeña y mediana empresa en España. Evolución reciente de los principales instrumentos públicos de apoyo, Boletín Económico de ICE, n. 2734, del 1 al 7 de julio, 2002, p. 23-32.

GARCÍA-GUTIÉRREZ-FERNÁNDEZ， C.: La reafirmación de las aportaciones de (propiedad de) los socios de las sociedades cooperativas. Propuesta de regulación de las sociedades de responsabilidad limitad cooperativa. REVESCO. Revista de Estudios Cooperativos, 2006, nº 89 , pp. 27-83.

GARCÍA-GUTIÉRREZFERNÁNDEZ, C.: El problema de la doble condición de sociostrabajadores (socios-proveedores y socios-consumidores) ante la gerencia de la empresa cooperativa, REVESCO. Revista de Estudios Cooperativos, 1987, n. 56-57.

ITURRIOZ DEL CAMPO, J.: Los procesos concursales en situación de crisis: características de su aplicación a las sociedades cooperativas. REVESCO. Revista de Estudios Cooperativos, 2010, $\mathrm{n}^{\circ}$ 100, pp. 134-159. 
ITURRIOZ DEL CAMPO, J.: El resultado en las sociedades cooperativas y su distribución en la nueva Ley de Cooperativas 27/1999, de 16 de julio. REVESCO, Revista de Estudios Cooperativos. n. 69, diciembre 1999.

ITURRIOZ DEL CAMPO, J.: La distribución de excedentes en las sociedades cooperativas ante el nuevo ordenamiento jurídico, Revista de Economía Pública, Social y Cooperativa CIRIEC-España n. 28, Madrid, junio de 1998.

ITURRIOZ DEL CAMPO, J. y MARTÍN LOPEZ, S.: Algunas especialidades financieras del concurso de acreedores de la sociedad cooperativa. Anuario de Derecho Concursal, $\mathrm{n}^{\mathrm{o}}$ 19, enero-abril 2010, p. 189-207.

MARTÍN LÓPEZ, S; LEJARRIAGA PÉREZ DE LAS VACAS, G. y GARCÍAGUTIÉRREZ FERNÁNDEZ, C.: Las dificultades de financiación de las empresas de participación ante la crisis económica: la creación de una entidad financiera de crédito como alternativa. REVESCO. Revista de Estudios Cooperativos, 2010, nº 100, pp. 11-44.

MARTÍN LÓPEZ, S., LEJARRIAGA PÉREZ DE LAS VACAS, G. e ITURRIOZ DEL CAMPO, J.: Consideraciones sobre la naturaleza del capital social en las sociedades cooperativas de trabajo asociado, REVESCO. Revista de Estudios Cooperativos, n. 91, 2007. p. 93-118.

MATEOS RONCO, A.: Los procesos concursales en sociedades cooperativas. Especificidades en la información económico-financiera. CIRIEC-España, Revista de Economía Pública, Social y Cooperativa, nº 60, 2008, p. 226-228.

SOTO ARANETA, M.: Consecuencias del concurso de acreedores para la empresa, Harvard Deusto Finanzas y Contabilidad, no.97, 2010, p. 4-12.

VARGAS VASSEROT, C.: La solvencia y garantías de las sociedades cooperativas en el tráfico. Algunas peculiaridades de su concurso, Revista de Derecho Concursal y Paraconcursal, n. 8, 2008, p. 287- 294.

\section{NORMATIVA LEGAL}

COMISIÓN EUROPEA. Recomendación 2003/361/CE de la Comisión, de 6 de mayo de 2003, sobre la definición de microempresas, pequeñas y medianas empresas [Diario Oficial L 124 de 20.5.2003].

ESPAÑA: LEY 27/1999, de 16 de julio, de Cooperativas, BOE. N. 170, de 17 de julio.

ESPAÑA: LEY ORGÁNICA 8/2003, de 9 de julio, para la reforma concursal, BOE N. 164, de 10 de julio. 
ESPAÑA: LEY 22/2003, de 9 de julio, Concursal, BOE, N. 164, de 10 de julio de 2003.

ESPAÑA. ORDEN EHA/3360/2010, de 21 de diciembre, por la que se aprueban las normas sobre los aspectos contables de las sociedades cooperativas. BOE, N. 316, de 29 de diciembre.

ESPAÑA: REAL DECRETO LEY 3/2009, de 27 de marzo, de medidas urgentes en materia tributaria, financiera y concursal ante la evolución de la situación económica, BOE N. 78, de 31 de marzo.

ESPAÑA: REAL DECRETO LEY 13/2009, de 3 de noviembre, de reforma de la legislación procesal para la implantación de la nueva Oficina Judicial, BOE N. 266, de 4 de noviembre. 